This is a postprint/Accepted version of the following published document:

Terán, F. de; Hernando, C. A class of quasi-sparse companion pencils. In: Structured matrices in numerical linear algebra: analysis, algorithms and applications. Cham, Switzerland: Springer Nature Switzerland AG, 2019, Pp. 157-179

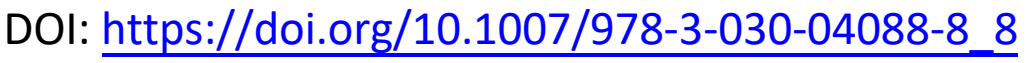

(c) Springer Nature Switzerland AG 2019. 


\title{
A class of quasi-sparse companion pencils
}

\author{
Fernando De Terán, Carla Hernando
}

\begin{abstract}
In this paper, we introduce a general class of quasi-sparse potential companion pencils for arbitrary square matrix polynomials over an arbitrary field, which extends the class introduced in [B. Eastman, I.-J. Kim, B. L. Shader, K.N. Vander Meulen, Companion matrix patterns. Linear Algebra Appl. 436 (2014) 255-272] for monic scalar polynomials. We provide a canonical form, up to permutation, for companion pencils in this class. We also relate these companion pencils with other relevant families of companion linearizations known so far. Finally, we determine the number of different sparse companion pencils in the class, up to permutation.
\end{abstract}

\section{Introduction}

The standard way to compute the eigenvalues and eigenvectors of a matrix polynomial

$$
Q(\lambda)=\sum_{i=0}^{k} \lambda^{i} A_{i}, \quad A_{i} \in \mathbb{F}^{n \times n}, \quad i=0,1, \ldots, k, \quad A_{k} \neq 0,
$$

(with $\mathbb{F}$ being an arbitrary field) is by means of a linearization, which is a matrix pencil (that is, a matrix polynomial of degree 1) whose eigenvalues (together with their multiplicities) coincide with the ones of the polynomial (1). Any matrix polynomial has infinitely many linearizations, but in order for them to be useful in practice, it is important to know in advance that they are linearizations. One way

Fernando De Terán

Departamento de Matemáticas, Universidad Carlos III de Madrid, Leganés, 28911, e-mail: fteranemath.uc3m.es.

Carla Hernando

Departamento de Matemáticas, Universidad Carlos III de Madrid, Leganés, 28911, e-mail: cahernanemath.uc3m.es 
to create such linearizations is by means of symbolic constructions consisting of block-partitioned pencils whose blocks contain the coefficients of (1). Companion pencils are particular cases of these constructions (see Definition 2.2). They present several advantages, besides being linearizations for any matrix polynomial. Among the most relevant ones are: (a) they are strong linearizations (that is, they also preserve the infinite eigenvalue of the polynomial, and its multiplicities), and (b) they present a template involving no arithmetic operations at all. The only information needed to build companion pencils is the selection and placement of the blocks.

Several families of companion pencils have been introduced in the literature, including the Fiedler-like families [1, 4, 8, 10, 23] and the block-Kronecker linearizations [15]. They contain, as particular cases, the classical Frobenius linearizations, and extend the notion of companion matrix, which has been extensively used to compute roots of scalar polynomials (like in the MATLAB command roots). In the recent years, some effort has been devoted to introduce new families of companion pencils which preserve some of the structures of matrix polynomials usually encountered in applications [2, 5, 7, 9], or to companion pencils in other polynomial bases than the monomial basis [20, 21, 22]. Some recent works have also analyzed particular features or applications of Fiedler-like pencils [3, 12, 13, 14]. In particular, it is proved in [6] that the families of Fiedler and Generalized Fiedler pencils are particular cases of block-Kronecker linearizations. As for companion matrices of scalar polynomials, we refer to [18] and [19] for a more general notion than the one considered in this paper, and to [17] for some pentadiagonal constructions.

Companion matrices are valid only for monic scalar polynomials. They have been studied in several recent papers [16, 18, 19] from a theoretical point of view, providing canonical expressions up to permutation. Some interest has also been paid to sparse companion matrices, namely those with the smallest number of nonzero entries, motivated by the simplicity of the constructions. However, from the numerical point of view, it may be desirable to work with non-monic polynomials. This is one of the motivations to introduce the more general notion of companion pencils.

In this paper, we are mainly interested in sparse companion pencils. Our main goal is to extend the results in [16] to such kind of constructions. In particular, we first introduce a general class of pencils (denoted by $\mathscr{R}_{n, k}$ ) associated with symbolic matrix polynomials as in (1). As with all families of companion pencils mentioned above, the pencils in $\mathscr{R}_{n, k}$ contain $k-1$ identity blocks, plus another $k-1$ blocks equal to $\lambda I$, together with some other nonzero blocks involving the coefficients of the polynomial. However, its generality relies on the fact that these blocks can be located anywhere in the pencil. This aims to introduce a class of potential linearizations that keeps all the essential structural properties of the previous families of linearizations (namely, the identity blocks), and having a small number of nonzero entries (or blocks). We refer to them as quasi-sparse because of this small number of nonzero entries. Some pencils in $\mathscr{R}_{n, k}$ can be either not companion (that is, linearizations) or not sparse, and our interest focuses on those which are companion (firstly) and those which are companion and sparse (secondly). Our goal is to provide a canonical expression, up to permutation, for companion pencils in this family, resembling the one provided in [16] for companion matrices, and to deter- 
mine, up to permutation as well, how many different sparse companion pencils are in this family. To achieve this goal, we introduce a new class of pencils, denoted by $\mathscr{Q} \mathscr{C}_{n, k}$ (plus an intermediate class $\mathscr{Q}_{n, k}$ ), which comprises, up to permutation, all companion pencils in $\mathscr{R}_{n, k}$, and we count the number of different sparse pencils in $\mathscr{Q} \mathscr{C}_{n, k}$.

The paper is organized as follows. In Section 2 we present the basic notions (including the families $\mathscr{R}_{n, k}, \mathscr{Q}_{n, k}$, and $\mathscr{Q}_{n, k}$ ), together with some structural properties. Section 3 is devoted to prove that any companion pencil in $\mathscr{R}_{n, k}$ is permutationally equivalent to a pencil in $\mathscr{Q}_{n, k}$, and that companion pencils in $\mathscr{Q}_{n, k}$ must belong to $\mathscr{Q} \mathscr{C}_{n, k}$. We also prove that all pencils in $\mathscr{Q}_{n, k}$ are companion pencils. In Section 4 we get the number of different sparse (companion) pencils in $\mathscr{Q} \mathscr{C}_{n, k}$.

\section{Preliminaries}

Throughout this paper, we use calligraphic letters with two subindices, like $\mathscr{A}_{n, k}$, to denote a class of $n k \times n k$ block-partitioned matrix pencils, which are viewed as block $k \times k$ matrices with blocks of size $n \times n$.

In order to define the notion of companion pencil for matrix polynomials, we first recall the following notions. For more information about them we refer to [11].

In what follows, the reversal of $Q(\lambda)$ in $(1)$ is the polynomial $\operatorname{rev} Q(\lambda):=$ $\sum_{j=0}^{k} \lambda^{j} A_{k-j}$, obtained by reversing the order of the coefficients of $Q(\lambda)$.

Definition 2.1. A matrix pencil $L(\lambda)=\lambda X+Y$ with $X, Y \in \mathbb{F}^{n k \times n k}$ is a linearization of an $n \times n$ matrix polynomial $Q(\lambda)$ of degree $k$ if there exist two unimodular $n k \times n k$ matrix polynomials $U(\lambda)$ and $V(\lambda)$ such that

$$
U(\lambda) L(\lambda) V(\lambda)=\left[\begin{array}{cc}
I_{(k-1) n} & 0 \\
0 & Q(\lambda)
\end{array}\right]
$$

(that is, $L(\lambda)$ is unimodularly equivalent to $\operatorname{diag}\left(I_{(k-1) n}, Q(\lambda)\right.$ ). The linearization is called $a$ strong linearization if $\operatorname{rev} L(\lambda)$ is also a linearization of $\operatorname{rev} Q(\lambda)$.

Definition 2.2. A companion pencil for general $n \times n$ matrix polynomials $\sum_{i=0}^{k} \lambda^{i} A_{i}$ of degree $k$ is an $n k \times n k$ matrix pencil $L(\lambda)=\lambda X+Y$ such that if $X$ and $Y$ are viewed as block $k \times k$ matrices with blocks of size $n \times n$, then:

(i) each nonzero block of $X$ and $Y$ is either $I_{n}$ or $A_{i}$ (up to constants), for some $i=0, \ldots, k$, and

(ii) $L(\lambda)$ is a strong linearization for every $n \times n$ matrix polynomial of degree $k$.

Note, in particular, that if $L(\lambda)$ is a companion pencil for $Q(\lambda)$, then $\operatorname{det}(L(\lambda))=$ $\alpha \operatorname{det}(Q(\lambda)$ ) (for some $\alpha \neq 0$ ). When $n=1, Q(\lambda)$ is just a scalar polynomial. In this case, we will use lowercase letters and this determinant condition reduces to $\operatorname{det}(L(\lambda))=\alpha q(\lambda)=\alpha \sum_{i=0}^{k} \lambda^{i} a_{i}$, with $0 \neq \alpha \in \mathbb{F}$. 


\subsection{New classes of block-partitioned pencils}

The most general family of "potential" companion pencils in this work, $\mathscr{R}_{n, k}$, is introduced in Definition 2.3 This family contains all the sparse companion pencils introduced so far in the literature (in the monomial basis). In particular both Fiedler and generalized Fiedler pencils [4, 8, 10], as well as the sparse block-Kronecker pencils introduced in [15, Def 5.1]. The motivation for introducing this family is precisely to create a general family containing all these companion pencils, and also to extend the family of companion matrices introduced in [16].

Definition 2.3. We denote by $\mathscr{R}_{n, k}$ the set of block-partitioned matrix pencils with block entries in $\mathbb{F}\left[A_{0}, \ldots, A_{k}\right]$ and whose only nonzero blocks are of the form:

- $k-1$ blocks equal to $-I$, together with $k-1$ blocks of the form $\lambda I$, and

- at most $k$ nonzero blocks, denoted by $B_{0}(\lambda), \ldots, B_{k-1}(\lambda)$, such that each coefficient $A_{i}$, for $i=0, \ldots, k$, appears only in one $B_{j}$, for $j=0, \ldots, k-1$. These blocks are of the form

$$
B_{j}(\lambda)=B_{j}^{0}+\lambda B_{j}^{1}
$$

for $j=0, \ldots, k-1$, with $B_{j}(\lambda)$ being either $0, A_{i}, \lambda A_{i+1}$ or $A_{i}+\lambda A_{i+1}$, for some $0 \leqslant i \leqslant k-1$.

The generality of the family $\mathscr{R}_{n, k}$ relies on the fact that nothing is said about the location of the nonzero blocks in Definition 2.3. Because of this generality, not all pencils in $\mathscr{R}_{n, k}$ are companion pencils, as we are going to see. The following subclass of $\mathscr{R}_{n, k}$ will comprise, up to permutation, all companion pencils of $\mathscr{R}_{n, k}$ (see Theorem 3.1).

Definition 2.4. $\mathscr{Q}_{n, k}$ is the class of block-partitioned pencils in $\mathscr{R}_{n, k}$ where:

- the blocks equal to -I are in all super-diagonal positions (i.e., the block entries $(i, i+1)$, for $i=1, \ldots, k-1)$,

- the blocks equal to $\lambda I$, together with a nonzero block $B_{d}$, for some $0 \leqslant d \leqslant k-1$, are on the main diagonal, and

- the remaining nonzero blocks $B_{j}$, for $j \neq d$, are below the main diagonal.

However, it is not difficult to see that not every matrix pencil in $\mathscr{Q}_{n, k}$ is a companion pencil [16, p. 261-262], since these pencils do not necessarily satisfy condition (ii) of Definition 2.2. The following result provides some necessary conditions in order for a pencil in $\mathscr{Q}_{n, k}$ to be companion.

Theorem 2.5. Let $L(\lambda) \in \mathscr{Q}_{n, k}$ be a companion pencil. Then,

(i) If $B_{j}$, for $1 \leqslant j \leqslant k-2$, is located in the ith subdiagonal, for $1 \leqslant i \leqslant k-2$, then $B_{j}$ is either $0, A_{k-i-1}, \lambda A_{k-i}$, or $A_{k-i-1}+\lambda A_{k-i}$.

(ii) If $B_{j}$ is located in the $(k-1)$ th subdiagonal, then $B_{j}$ is either $A_{0}$ or $A_{0}+\lambda A_{1}$.

(iii) If $B_{j}$ is located on the main diagonal, then $B_{j}$ is either $\lambda A_{k}$ or $\lambda A_{k}+A_{k-1}$.

In order to prove Theorem 2.5 we use the following result. 
Lemma 2.6. Let $L(\lambda)=\left[l_{i j}\right] \in \mathscr{Q}_{1, k}$. For any nonzero $l_{s t}$ with $s-t \geqslant 0$, the determinant of $L(\lambda)$ contains a nonzero summand of the form:

$$
l_{11} \cdots l_{t-1, t-1} l_{s t} l_{s+1, s+1} \cdots l_{k k}
$$

Proof. Spanning either across the row or the column containing $l_{s t}$, for some $s-t \geqslant$ 0 , we obtain that the only term in $\operatorname{det}(L(\lambda))$ containing $l_{s t}$ is $l_{s t} C_{s t}$, where $C_{s t}$ is the cofactor of the block entry $l_{s t}$. This cofactor is of the form:

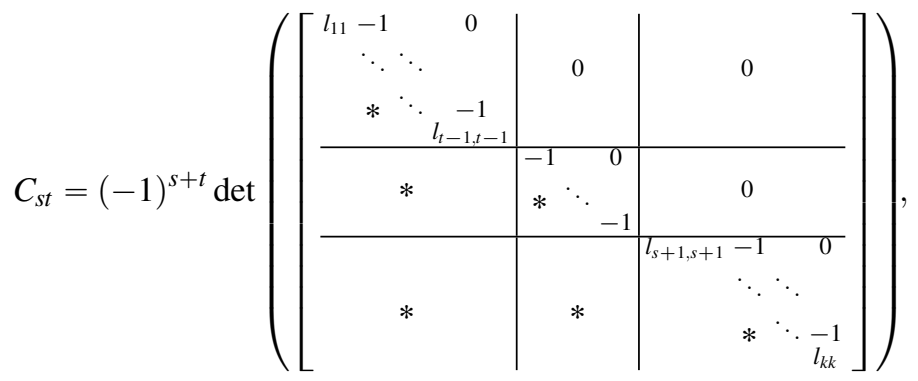

that is, $C_{s t}=l_{11} \cdots l_{t-1, t-1} l_{s+1, s+1} \cdots l_{k k}+\widetilde{C}_{s t}$. Recall that below the main diagonal of $L(\lambda)$ there can be nonzero entries. Since $L(\lambda) \in \mathscr{Q}_{1, k}$, the first summand in $C_{s t}$ has degree $k-(s-t)-1$. It suffices to prove that $\widetilde{C}_{s t}$ has, at most, degree $k-(s-t)-2$.

First, the matrix in (4) is partitioned in six big blocks. Note that each summand in $\widetilde{C}_{s t}$ contains a term below the main diagonal multiplied by its cofactor, in particular, this term can be in one of the $(1,1),(2,1),(2,2),(3,1),(3,2)$ or $(3,3)$ blocks. If it is in any of the blocks $(2,1),(2,2)$ or $(3,2)$, its cofactor is 0 . However, if it is in the remaining blocks $(1,1),(3,1)$ or $(3,3)$, its cofactor is obtained by removing two terms on the main diagonal, which are of degree 1 in $\lambda$, and the cofactor is multiplied by the term on the subdiagonal, which is, at most, of degree 1 in $\lambda$. Then, $\widetilde{C}_{s t}$ is, at most, of degree $k-(s-t)-2$ in $\lambda$.

Finally, by Definition 2.3. $l_{s t}$ contains some coefficient $a_{r}$, which does not appear in any other entry, so (3) cannot cancel out with any other term in $\operatorname{det}(L(\lambda))$.

Proof (Theorem 2.5). We focus on $n=1$. Let $L(\lambda) \in \mathscr{Q}_{1, k}$. If $a_{i}$, for $0 \leqslant i \leqslant k$, is in $l_{s t}$ (in the $r$ th subdiagonal, with $r:=s-t$ ), then the exponent of $\lambda$ which appears multiplied by $a_{i}$ in $\operatorname{det}(L(\lambda))$ is, by (3), equal to $k-r-1+\operatorname{deg}\left(l_{s t}\right)$ (note that $l_{11}, \ldots, l_{t-1, t-1}, l_{s+1, s+1}, \ldots, l_{k k}$ all have degree 1$)$. Then, $i=k-r-1+\operatorname{deg}\left(l_{s t}\right)$, so $r=k-i-1+\operatorname{deg}\left(l_{s t}\right)$, but $\operatorname{deg}\left(l_{s t}\right)$ is either 0 or 1 , and $a_{i}$ must be either in the $(k-i-1)$ th subdiagonal (without $\lambda$ ) or in the $(k-i)$ th subdiagonal (multiplied by $\lambda$ ). In particular, when $i=0$, the only possibility is $\operatorname{deg}\left(l_{s t}\right)=0$ and $r=k-1$ (otherwise, if $\operatorname{deg}\left(l_{s t}\right)=1$, we would have $r=k$, which is not possible, since there are no $k$ subdiagonals), and similarly when $i=k$, the only possibility is $\operatorname{deg}\left(l_{s t}\right)=1$ and $r=0$. This means that $a_{0}$ can only be in the $(k-1)$ th subdiagonal (without $\lambda$ ) and $a_{k}$ can only be in the 0th subdiagonal (multiplied by $\lambda$ ).

As a consequence, if $b_{j}=b_{j}^{0}+\lambda b_{j}^{1}$ is in the $i$ th subdiagonal, then $b_{j}^{0}$ can be either 0 or $a_{k-i-1}$, and $b_{j}^{1}$ can be either 0 or $a_{k-i}$. 
Now, we introduce the following class of block-partitioned pencils where part $(i)$ is motivated by Theorem 2.5 .

Definition 2.7. $\mathscr{Q} \mathscr{C}_{n, k}$ is the class of block-partitioned pencils in $\mathscr{Q}_{n, k}$ satisfying the following conditions:

(i) The coefficient $A_{i}$ is either in the $(k-i-1)$ th subdiagonal or in the $(k-i)$ th subdiagonal. In the first case it appears without $\lambda$, and in the second one it appears multiplied by $\lambda$.

(ii) (Rectangle condition). All possible nonzero blocks $B_{j}$, for $j=0,1, \ldots, k-1$, lie on the rectangular block-partitioned submatrix whose upper right corner is the position containing $A_{k}$, which is on the main diagonal (denoted as $B_{k-1}$ ), and whose lower left corner is the position containing $A_{0}$ (denoted by $B_{0}$ ), namely the $(k, 1)$ position.

The following example illustrates the difference between Definitions 2.4 and 2.7 .

Example 2.8. Let $Q(\lambda)=\sum_{i=0}^{4} \lambda^{i} A_{i}$ be an $n \times n$ matrix polynomial of degree 4 . Let us consider the following block-partitioned matrix pencils $L_{1}(\lambda)$ and $L_{2}(\lambda)$.

$$
L_{1}(\lambda)=\left[\begin{array}{cccc}
\lambda I & -I & 0 & 0 \\
0 A_{3}+\lambda A_{4} & -I I & 0 \\
0 & 0 & -1 \\
A_{0} & A_{1} & A_{2} & \lambda I
\end{array}\right] \text { and } L_{2}(\lambda)=\left[\begin{array}{cccc}
\lambda I & -I & 0 & 0 \\
A_{2}+\lambda A_{3} & \lambda A_{4} & -I & 0 \\
0 & 0 & \lambda I & -I \\
A_{0}+\lambda A_{1} & 0 & 0 & \lambda I
\end{array}\right] .
$$

In $L_{1}(\lambda)$, the coefficient $A_{2}$ is not inside the rectangular block-partitioned submatrix indicated with a box. In $L_{2}(\lambda)$, instead, all nonzero blocks below the main diagonal are in this rectangle. Then, $L_{1}(\lambda) \in \mathscr{Q}_{n, 4} \backslash \mathscr{Q} \mathscr{C}_{n, 4}$, and $L_{2}(\lambda) \in \mathscr{Q}_{n, 4}$.

Figure 1 illustrates the relationship between the classes $\mathscr{R}_{n, k}, \mathscr{Q}_{n, k}$, and $\mathscr{Q}_{n, k}$.

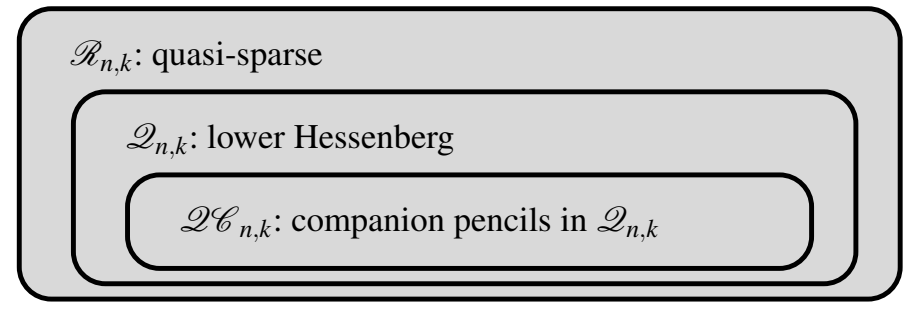

\section{Companion pencils in $\mathscr{Q}_{n, k}$}

Our first result shows that any companion pencil in $\mathscr{R}_{n, k}$ can be taken to the form $\mathscr{Q}_{n, k}$ by block permutations of rows and columns.

Theorem 3.1. Any companion pencil in $\mathscr{R}_{n, k}$ is block permutationally equivalent to a pencil in $\mathscr{Q}_{n, k}$.

Proof. We can focus on the case $n=1$ for simplicity. All developments are also true for arbitrary $n$. 
Let $S(\lambda) \in \mathscr{R}_{1, k}$ be a companion pencil for the scalar polynomial $q(\lambda)=\sum_{i=0}^{k} \lambda^{i} a_{i}$, which has $k-1$ entries equal to -1 , together with $k-1$ entries equal to $\lambda$ and, at most, $k$ nonzero entries that we order as $b_{0}, \ldots, b_{k-1}$. The polynomials $b_{j}$, for $j=0, \ldots, k-1$, are equal to either $0, a_{i}, \lambda a_{i+1}$, or $a_{i}+\lambda a_{i+1}$, for some $0 \leqslant i \leqslant k-1$, as in Definition 2.3 Suppose, $b_{k-1}$ is the entry containing $a_{k}$ and $b_{0}$ is the one containing $a_{0}$. Then, $b_{k-1}$ and $b_{0}$ must be of the form

$$
b_{k-1}(\lambda)=\left\{\begin{array}{c}
\lambda a_{k}, \text { or } \\
a_{k-1}+\lambda a_{k},
\end{array} \text { and } b_{0}(\lambda)=\left\{\begin{array}{c}
a_{0}, \text { or } \\
a_{0}+\lambda a_{1} .
\end{array}\right.\right.
$$

Since $S(\lambda)$ is a companion pencil, $\operatorname{det}(S(\lambda))=\alpha q(\lambda)=\alpha \sum_{i=0}^{k} \lambda^{i} a_{i}$, with $0 \neq \alpha \in$ $\mathbb{F}$ (note that, since the leading term of $\operatorname{det}(S(\lambda))$ comes from the product of the $k-1$ entries equal to $\lambda$, together with $b_{k-1}$, it must be $\alpha= \pm 1$ ). This identity is satisfied for all values of the coefficients $a_{i}$. Then, we can shrink to zero some coefficients $a_{i}$ or give them some specific values and the identity, for these particular values, must be true as well.

In the first place, we shrink to zero all the coefficients $a_{i}$ of $q(\lambda)$ which are not in $b_{k-1}$, that is, we assume that all entries $b_{j}$ are zero except $b_{k-1}$. In this case, $\operatorname{det}(S(\lambda))=\alpha\left(\lambda^{k-1} b_{k-1}\right)$. This implies that all entries equal to $\lambda$, together with $b_{k-1}$, are in different rows and columns of $S(\lambda)$.

Similarly, by shrinking to zero all the coefficients $a_{i}$ of $q(\lambda)$ which are not in $b_{0}$, we conclude that all entries equal to -1 , together with $b_{0}$, are in different rows and columns of $S(\lambda)$.

Now, we can find two permutation matrices $P_{1}, P_{2}$ such that

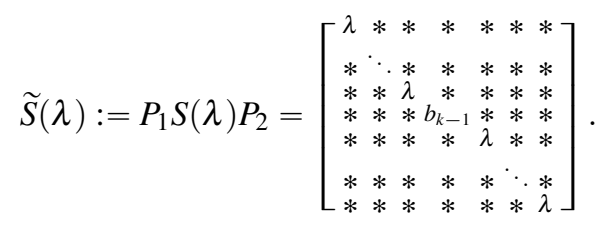

To be precise, $P_{2}$ is built up as follows: $S(\lambda)$ has, in each row, only one element equal to either $\lambda$ or $b_{k-1}$. Then, we can define $P_{2}$ as the matrix that takes this element, which is in the position $\left(i, j_{i}\right)$, to the position $(i, i)$, for each $i=1, \ldots, k$.

Similarly, we can proceed with columns instead of rows to define $P_{1}$. Then, we only need one of $P_{1}$ or $P_{2}$, depending on whether we perform row or column permutations. Therefore, up to permutational equivalence, we get the pencil $\widetilde{S}(\lambda)$, with the same entries as $S(\lambda)$, but with the $k-1$ entries equal to $\lambda$, together with $b_{k-1}$, on the main diagonal.

There are, at most, $2(k-1)$ nonzero entries $(*)$ in $\widetilde{S}(\lambda)$, which are the $k-1$ entries equal to -1 together with the polynomials $b_{j}$, for $j=0, \ldots, k-2$. Note that $b_{k-1}$ can be in any position on the main diagonal, and that the $k-1$ entries equal to -1 , together with $b_{0}$, are also in different rows and columns. We are going to show that there is a permutation matrix $\widetilde{P}_{1}$ such that 


$$
\widehat{S}(\lambda):=\widetilde{P}_{1} \widetilde{S}(\lambda) \widetilde{P}_{1}^{T}=\left[\begin{array}{ccccccc}
\lambda & -1 & * & * & * & * & * \\
* & \ddots & \ddots & * & * & * & * \\
* & * & \lambda & -1 & * & * & * \\
* & * & * & b_{k-1} & -1 & * & * \\
* & * & * & * & \lambda & \ddots & * \\
* & * & * & * & * & \ddots & -1 \\
* & * & * & * & * & * & \lambda
\end{array}\right]
$$

Note that the entries equal to $\lambda$ and $b_{k-1}$ remain on the main diagonal in $\widehat{S}(\lambda)$.

It suffices to prove that the $k$ entries $\left(-1\right.$ 's and $\left.b_{0}\right)$ form a $k$-cycle. For this, let us shrink to zero all coefficients $a_{i}$ of $q(\lambda)$ other than $a_{0}$ and $a_{k}$, set $a_{k}=1$, and denote by $\widetilde{S}_{1}(\lambda)$ the pencil obtained from $\widetilde{S}(\lambda)$ after this replacement. Then $\operatorname{det}\left(\widetilde{S}_{1}(\lambda)\right)=\lambda^{k}+a_{0}$. Moreover, $\widetilde{S}_{1}(\lambda)$ does not contain any other terms with degree 1 in $\lambda$ than the ones on the main diagonal, so $\widetilde{S}_{1}(\lambda)=\lambda I-A$, with $A$ being a companion matrix for the polynomial $\lambda^{k}+a_{0}$.

As a consequence of Lemma 2.1 in [16], we conclude that the -1 entries, together with the one containing $a_{0}$, must be in a cycle of length $k$.

Therefore, up to permutational similarity, we arrive at $\widehat{S}(\lambda)$, having the same entries as $\widetilde{S}(\lambda)$, but the $k-1$ entries equal to -1 being on the super-diagonal and the entry $b_{0}$ being in the position $(k, 1)$.

Finally, let us assume, by contradiction, that $\widehat{S}(\lambda)$ has, at least, one entry $b_{t}$, for some $1 \leqslant t \leqslant k-2$, above the super-diagonal, that is, in the position $\left(i_{t}, j_{t}\right)$, with $i_{t}<j_{t}-1$.

Let us shrink to zero all the coefficients $a_{i}$ of $q(\lambda)$ which are not in $b_{t}$ and denote by $\widehat{S}_{1}(\lambda)$ the pencil obtained after this replacement. Note that either $b_{t}(\lambda)=a_{r}$, $b_{t}(\lambda)=\lambda a_{r+1}$, or $b_{t}(\lambda)=a_{r}+\lambda a_{r+1}$, for some $1 \leqslant r \leqslant k-2$. We suppose that $b_{t}(\lambda)=a_{r}+\lambda a_{r+1}$ (the proof is analogous for the other two cases). In this case, since $\hat{S}(\lambda)$ is a companion pencil, it must be $\operatorname{det}\left(\hat{S}_{1}(\lambda)\right)=\lambda^{r} a_{r}+\lambda^{r+1} a_{r+1}$, where

$$
\widehat{S}_{1}(\lambda):=\widehat{S}\left(0, \ldots, 0, a_{r}, a_{r+1}, 0, \ldots, 0 ; \lambda\right)=\left[\begin{array}{ccccccc}
\lambda & -1 & & & & & \\
& \ddots & \ddots & & & & \\
& \lambda & \ddots & & & \\
& & & \\
& & 0 & \ddots & \\
& & & \lambda & \ddots & \\
& & & & \ddots & -1 \\
& & & & & \lambda
\end{array}\right] .
$$

This matrix is upper triangular and its determinant is the product of the entries on the main diagonal, that is, $\operatorname{det}\left(\widehat{S}_{1}\right)=0 \neq \lambda^{r} a_{r}+\lambda^{r+1} a_{r+1}$, which is a contradiction. Therefore, all nonzero entries in $\widehat{S}(\lambda)$ are on or below the super-diagonal.

Next we prove that any companion pencil in $\mathscr{Q}_{n, k}$ belongs to $\mathscr{Q} \mathscr{C}_{n, k}$.

Theorem 3.2. Let $L(\lambda) \in \mathscr{Q}_{n, k}$ be a companion pencil. Then, $L(\lambda) \in \mathscr{Q} \mathscr{C}_{n, k}$.

Proof. As in the proof of Theorem 3.1, we can focus on the case $n=1$. Then, let $L(\lambda) \in \mathscr{Q}_{1, k}$. By Theorem 2.5. we know that if the polynomial $b_{j}$, for $j=1, \ldots, k-2$, is in the $i$ th subdiagonal of $L(\lambda)$, for $i=1, \ldots, k-2$, then $b_{j}$ is either $0, a_{k-i-1}$, 
$\lambda a_{k-i}$, or $a_{k-i-1}+\lambda a_{k-i}$. Therefore, it satisfies condition (i) in Definition 2.7 It remains to prove that $L(\lambda)$ satisfies condition (ii) in Definition 2.7.

By contradiction, let us assume that there is an entry $b_{t}$, for some $1 \leqslant t \leqslant k-2$, outside the rectangle. Then it is located in the position $\left(i_{t}, j_{t}\right)$, with either $j_{t}<i_{t}<i_{0}$ or $i_{t}>j_{t}>i_{0}$, where $l_{i_{0}, i_{0}}(\lambda)=b_{k-1}(\lambda)$. Let us shrink to zero all the coefficients $a_{i}$ of the polynomial $q(\lambda)$ which are not in $b_{t}$ or $b_{k-1}$ and let us denote by $L_{1}(\lambda)$ the pencil obtained after doing this. Then, $L_{1}(\lambda)$ is of the form

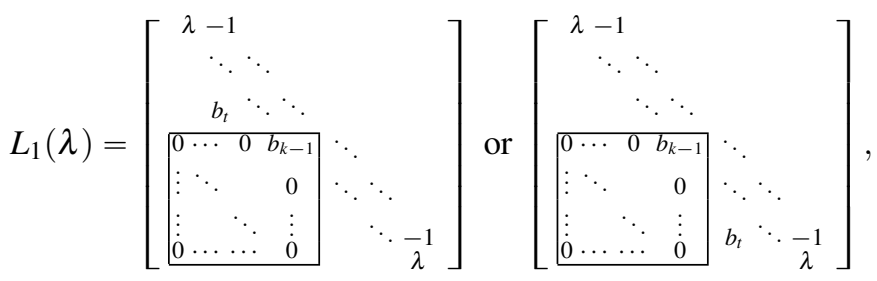

depending on whether $j_{t}<i_{t}<i_{0}$ or $i_{t}>j_{t}>i_{0}$. In both cases, the entries not explicitly indicated in the matrix are zero. Let us first assume that $j_{t}<i_{t}<i_{0}$.

By Lemma 2.6, the determinant of $L_{1}(\lambda)$ will contain a nonzero term of the form:

$$
\underbrace{l_{11} \cdots l_{j_{t}-1, j_{t}-1}}_{\lambda^{\prime} s} \underbrace{l_{i_{t}, j_{t}}}_{b_{t}} \cdot \underbrace{l_{i_{t}+1, i_{t}+1} \cdots l_{k k}}_{\lambda^{\prime} \text { s and } b_{k-1}}=\lambda^{k-\left(i_{t}-j_{t}\right)-2} b_{t} b_{k-1} .
$$

Therefore, $\operatorname{det}\left(L_{1}(\lambda)\right)$ contains a term involving the product $b_{t} b_{k-1}$, which involves in turn a product of coefficients $a_{i}$ of $q(\lambda)$, and this is in contradiction with the fact that $L(\lambda)$ is a companion pencil.

It remains to analyze the case where $i_{0}<j_{t}<i_{t}$. The determinant of $L_{1}(\lambda)$ for this case contains a nonzero term of the form:

$$
\underbrace{l_{11} \cdots l_{j_{t}-1, j_{t}-1}}_{\lambda^{\prime} s \text { and } b_{k-1}} \cdot \underbrace{l_{i_{t}, j_{t}}}_{b_{t}} \cdot \underbrace{l_{i_{t}+1, i_{t}+1} \cdots l_{k k}}_{\lambda^{\prime} s}=\lambda^{k-\left(i_{t}-j_{t}\right)-2} b_{t} b_{k-1} .
$$

As above, this is a contradiction with the fact that $L(\lambda)$ is a companion pencil.

Theorem 3.2 tells us that a matrix pencil in $\mathscr{Q}_{n, k}$ must belong to $\mathscr{Q} \mathscr{C}_{n, k}$ in order to be a companion pencil. The following result shows that, moreover, all pencils in $\mathscr{Q} \mathscr{C}_{n, k}$ are companion.

Theorem 3.3. Any pencil in $\mathscr{Q} \mathscr{C}_{n, k}$ is a companion pencil.

Proof. Let $L(\lambda) \in \mathscr{Q} \mathscr{C}_{n, k}$ be an $n k \times n k$ matrix pencil. If $B_{k-1}$ is in the entry $(p+$ $1, p+1)$, for some $0 \leqslant p \leqslant k-1$, then we can write $L(\lambda)$ as the following blockpartitioned matrix pencil: 


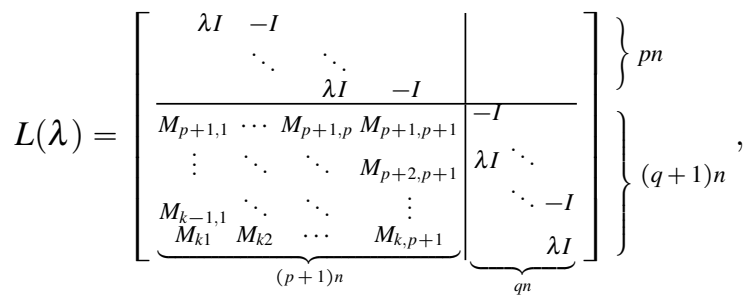

with $p+q+1=k$. Note that $M_{k 1}=B_{0}, M_{p+1, p+1}=B_{k-1}$, and the remaining blocks $M_{s t}$, for $s=p+1, \ldots, k$ and $t=1, \ldots, p+1$, are either 0 or $B_{j}$, for $j=1, \ldots, k-2$. Note that, by Theorem 2.5, if $B_{j}$, for $j=1, \ldots, k-2$, is in the $r$ th subdiagonal of $L(\lambda)$, for $r=1, \ldots, k-2$, then it is either $0, A_{k-r-1}, \lambda A_{k-r}$, or $A_{k-r-1}+\lambda A_{k-r}$.

Now, we consider the following two block permutations $P_{\text {row }}$ and $P_{\text {col }}$ :

- $P_{\text {row }}$ permutes the rows of $L(\lambda)$. Note that $L(\lambda)$ is partitioned in two big blocks of block-partitioned matrices by rows; the first block-partitioned matrix includes rows from 1 to $p$ and the second one includes rows from $p+1$ to $k$. We define $P_{\text {row }}$ as the matrix taking: $s \mapsto k-s+1$, for $s=1, \ldots, p$, and $s \mapsto s-p$, for $s=p+1, \ldots, k$.

- $P_{\text {col }}$ permutes only the first $p+1$ columns of the matrix $L(\lambda)$. We define $P_{\text {col }}$ as the matrix taking: $t \rightarrow p+2-t$, for $t=1, \ldots, p+1$.

It is straightforward to see that:

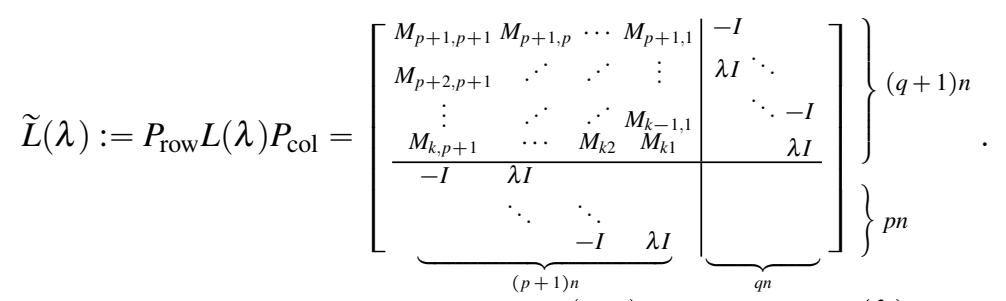

In particular, the (block) diagonals in the $(2,1)$ big block of $L(\lambda)$ become the (block) anti-diagonals of the $(1,1)$ big block in $\widetilde{L}(\lambda)$. Now, let us consider a general pencil like in the right-hand side of (5). In particular, the blocks $M_{s t}$ are matrix pencils. It is shown in [15, Thm. 5.4] that if the sum of the trailing coefficients of all $M_{s t}$ blocks in the $(k-i-1)$ th antidiagonal plus the sum of the leading coefficients of all $M_{s t}$ blocks in the $(k-i)$ th antidiagonal equals $A_{i}$, for all $i=0,1, \ldots, k$, then $\widetilde{L}(\lambda)$ is a strong linearization of $Q(\lambda)$. By condition (i) in Definition 2.7, this condition on the leading and trailing coefficients of the $M_{s t}$ blocks is satisfied for the particular $\widetilde{L}(\lambda)$ coming from $L(\lambda)$ as in $(5)$. Therefore, $\widetilde{L}(\lambda)$ is a strong linearization of $Q(\lambda)$. Since $L(\lambda)$ is permutationally equivalent to $\widetilde{L}(\lambda), L(\lambda)$ is also a strong linearization of $Q(\lambda)$. Then, $L(\lambda)$ satisfies condition (ii) in Definition 2.2 and, by definition, $L(\lambda)$ satisfies condition (i) as well, so it is a companion pencil.

The proof of Theorem 3.3 shows that the family of block-Kronecker pencils introduced in [15, Def. 5.1] comprise, up to block permutation, all companion pencils in $\mathscr{R}_{n, k}$ (in other words, it contains, up to block permutation, all pencils in $\mathscr{Q} \mathscr{C}_{n, k}$ ). 


\section{Number of different sparse companion pencils in $\mathscr{R}_{n, k}$}

We say that companion pencils in $\mathscr{R}_{n, k}$ are quasi-sparse companion pencils, since they have a small number of nonzero block entries. However, not all companion pencils in $\mathscr{R}_{n, k}$ have the same number of nonzero block entries. We first give a lower bound on the number of nonzero block entries of a companion pencil in $\mathscr{R}_{n, k}$. In the following, $\lfloor r\rfloor$ and $\lceil r\rceil$ denote, respectively, the floor and the ceiling of $r \in \mathbb{R}$. Note that, if $r$ is an integer, then $r=\lfloor r\rfloor=\lceil r\rceil$.

Lemma 4.1. Any companion pencil in $\mathscr{R}_{n, k}$ has, at least, $2 k-1+\left\lfloor\frac{k}{2}\right\rfloor$ nonzero block entries.

Proof. By Theorems 3.1 and 3.2 , any companion pencil in $\mathscr{R}_{n, k}$ is permutationally equivalent to a pencil in $\mathscr{Q} \mathscr{C}_{n, k}$, so we focus on pencils in $\mathscr{Q} \mathscr{C}_{n, k}$. Recall (Theorem 2.5) that the blocks $B_{j}$, for $j=0, \ldots, k-1$, are equal to either $0, A_{i}, \lambda A_{i+1}$, or $A_{i}+\lambda A_{i+1}$, for some $0 \leqslant i \leqslant k-1$. Note that the entries equal to $-I$ and $\lambda I$ add up to $2(k-1)$ nonzero block entries. Regarding the $k+1$ coefficients $A_{i}(i=0, \ldots, k)$, they can be grouped in the pencils $B_{j}=B_{j}^{0}+\lambda B_{j}^{1}(j=0, \ldots, k-1)$. If $k$ is odd, then $\frac{k+1}{2}=\left\lceil\frac{k+1}{2}\right\rceil$ is the smallest number of nonzero blocks $B_{j}$, which are of the form $A_{2 i}+\lambda A_{2 i+1}$, for $i=0,1, \ldots, \frac{k-1}{2}$. However, if $k$ is even, different groupings are possible, but in all cases the smallest number of nonzero blocks $B_{j}$ is $\left\lfloor\frac{k+1}{2}\right\rfloor+1=$ $\left\lceil\frac{k+1}{2}\right\rceil$. Adding up, we get $2(k-1)+\left\lceil\frac{k+1}{2}\right\rceil=2 k-1+\left\lfloor\frac{k}{2}\right\rfloor$ nonzero block entries.

Lemma 4.1 motivates the following definition.

Definition 4.2. A sparse pencil in $\mathscr{R}_{n, k}$ is a pencil with exactly $2 k-1+\left\lfloor\frac{k}{2}\right\rfloor$ nonzero block entries.

Since any companion pencil in $\mathscr{R}_{n, k}$ is permutationally equivalent to a pencil in $\mathscr{Q} \mathscr{C}_{n, k}$, to count the number of sparse companion pencils in $\mathscr{R}_{n, k}$, up to permutation, we can just count the number of non-permutationally equivalent pencils in $\mathscr{Q} \mathscr{C}_{n, k}$. First, Theorem 4.3 guarantees that no two of them are permutationally equivalent.

Theorem 4.3. Two different matrix pencils in $\mathscr{Q C}_{n, k}$ are not block permutationally equivalent.

Proof. Let $L_{1}(\lambda), L_{2}(\lambda) \in \mathscr{Q} \mathscr{C}_{n, k}$. If $L_{1}(\lambda)$ is block permutationally equivalent to $L_{2}(\lambda)$ there exists two block-partitioned permutation matrices $P, P^{\prime}$ with

$$
P L_{1}(\lambda) P^{\prime}=L_{2}(\lambda)
$$

Let us shrink to zero all coefficients $A_{i}$, for $i=0, \ldots, k-1$, and let $A_{k}=I$. Looking only at the leading terms in (6), we get $P \cdot I \cdot P^{\prime}=I$, so $P=\left(P^{\prime}\right)^{-1}$. Then, $L_{1}(\lambda)$ and $L_{2}(\lambda)$ are block permutationally similar.

It suffices to prove that two different matrix pencils in $\mathscr{Q} \mathscr{C}_{n, k}$ are not block permutationally similar. $L_{1}(\lambda)$ is block permutationally similar to $L_{2}(\lambda)$ if there exists a block-partitioned permutation matrix $P$ such that 


$$
P L_{1}(\lambda) P^{B}=L_{2}(\lambda)
$$

(where $(\cdot)^{B}$ stands for block transposition). We prove that the only permutation matrix $P$ satisfying (7) is the identity matrix, which implies $L_{1}(\lambda)=L_{2}(\lambda)$. For this, we can focus on the case $n=1$.

Again, by shrinking to zero all entries $a_{i}$, for $i=0, \ldots, k$, and equating the trailing coefficients in (7) we get $P N=N P$, with

$$
N=\left[\begin{array}{cccc}
0 & 1 & & \\
& \ddots & \ddots & \\
& & 0 & 1 \\
& & & 0
\end{array}\right] .
$$

It is straightforward to check that the only permutation matrix $P$ with $P N=N P$ is $P=I$.

The following lemmas will help us to get the number of sparse pencils in $\mathscr{Q} \mathscr{C}_{n, k}$.

Lemma 4.4. If $L(\lambda) \in \mathscr{Q} \mathscr{C}_{n, k}$, then $L(\lambda)$ cannot have two consecutive null subdiagonals.

Proof. Just recall, by condition (i) in Definition 2.7 that $A_{k-j}$ is either in the $(j-$ 1)th subdiagonal or in the $j$ th subdiagonal (as $\lambda A_{k-j}$ ), for $j=1, \ldots, k-2$.

Lemma 4.5. If $L(\lambda) \in \mathscr{Q}_{n, k}$ is sparse, then it can not have two nonzero block entries in the same subdiagonal.

Proof. If $L(\lambda) \in \mathscr{Q} \mathscr{C}_{n, k}$ is sparse and it has two nonzero block entries in the $j$ th subdiagonal, for some $1 \leqslant j \leqslant k-2$, these must be $A_{k-j-1}$ and $\lambda A_{k-j}$ (by Definitions 2.3 and 2.7). Then, by joining $A_{k-j-1}+\lambda A_{k-j}$ in the same entry (either the one containing $A_{k-j-1}$ or the one containing $\lambda A_{k-j}$ ) we arrive at a new pencil in $\mathscr{Q} \mathscr{C}_{n, k}$ having less nonzero block entries than $L(\lambda)$, which is a contradiction with the fact that $L(\lambda)$ is sparse.

The following result gives us the exact number of zero subdiagonals (including the main diagonal as the 0th subdiagonal) of any sparse pencil in $\mathscr{Q}_{n, k}$.

Lemma 4.6. Let $L(\lambda) \in \mathscr{Q}_{n, k}$ be sparse. Then $L(\lambda)$ has exactly $k-1-\left\lfloor\frac{k}{2}\right\rfloor$ null subdiagonals.

Proof. If $L(\lambda) \in \mathscr{Q} \mathscr{C}_{n, k}$ is sparse, it has $\left\lfloor\frac{k}{2}\right\rfloor$ nonzero entries below the diagonal, by Lemma 4.1. By Lemma 4.5. no two nonzero entries of $L(\lambda)$ are in the same subdiagonal, so $L(\lambda)$ has $\left\lfloor\frac{k}{2}\right\rfloor$ nonzero subdiagonals and, as a consequence, $k-1-$ $\left\lfloor\frac{k}{2}\right\rfloor$ null subdiagonals.

As a consequence of the previous results, we can explicitly identify which are the only null subdiagonals of any sparse pencil in $\mathscr{Q} \mathscr{C}_{n, k}$. This is the first step in determining the number of sparse pencils in $\mathscr{Q} \mathscr{C}_{n, k}$. We start with the case $k$ odd.

Lemma 4.7. Let $L(\lambda) \in \mathscr{Q}_{n, k}$ be a sparse pencil with $k$ odd. Then the only nonzero $j$ th subdiagonals of $L(\lambda)$ are the ones with indices $j=0,2,4, \ldots, k-1$. 
Proof. By Lemma 4.6. $L(\lambda)$ has exactly $\frac{k-1}{2}$ null subdiagonals and, consequently, $\frac{k+1}{2}$ nonzero subdiagonals. Since the main diagonal and the $(k-1)$ st subdiagonal (the entry $(k, 1)$ ) are nonzero, among the remaining $k-2$ subdiagonals there are $\frac{k-1}{2}$ null ones, together with another $\frac{k-3}{2}$ nonzero ones. Since, by Lemma 4.4 there can not be two consecutive null subdiagonals in $L(\lambda)$, the first and the $(k-2)$ nd subdiagonal must be zero, and the zero/nonzero subdiagonals must alternate.

For $k$ even, the situation is more involved. As there are $k+1$ coefficients $A_{i}$, for $i=0, \ldots, k$, there must be $\frac{k}{2}$ nonzero blocks of the form $A_{k-j-1}+\lambda A_{k-j}$, together with another nonzero block of the form $B_{\ell}=\lambda A_{k-j}$ or $B_{\ell}=A_{k-j-1}$, for $0 \leqslant j \leqslant$ $k-1$. In particular, the pattern of zero/nonzero subdiagonals in $\mathscr{Q} \mathscr{C}_{n, k}$ depends on the position of this monomial $B_{\ell}$. This is stated in Lemma 4.8, which also establishes some restrictions about $B_{\ell}$.

Lemma 4.8. Let $L(\lambda) \in \mathscr{Q}_{n, k}$ be a sparse pencil with $k$ even. Then the monomial $B_{\ell}$, located in the $j$ th subdiagonal (for $0 \leqslant j \leqslant k-1$ ), and the indices of the nonzero rth subdiagonals of $L(\lambda)$ are the following:

(a) If $j$ is even: $B_{\ell}=\lambda A_{k-j}$, and $r=0,2,4, \ldots, j, j+1, j+3, \ldots, k-3, k-1$;

(b) If $j$ is odd: $B_{\ell}=A_{k-j-1}$, and $r=0,2,4, \ldots, j-1, j, j+2, \ldots, k-3, k-1$.

Proof. By Lemma $4.6, L(\lambda)$ has exactly $\frac{k}{2}-1$ zero subdiagonals and, consequently, $\frac{k}{2}+1$ nonzero subdiagonals. Recall that the main diagonal and the $(k-1)$ th subdiagonal (the entry $(k, 1)$ ) are nonzero. As mentioned above, there is only one nonzero block of the form $B_{\ell}=\lambda A_{k-j}$ or $A_{k-j-1}$, which is located in the $j$ th subdiagonal, for some $0 \leqslant j \leqslant k-1$. The remaining nonzero blocks are of the form $A_{k-s-1}+\lambda A_{k-s}$, for $s \neq j$. As a consequence, there are four possible situations:

Case 1: $B_{\ell}=\lambda A_{k-j}$ is in the $j$ th subdiagonal, for $0 \leqslant j \leqslant k-1$, with $j$ even.

Case 2: $B_{\ell}=\lambda A_{k-j}$ is in the $j$ th subdiagonal, for $0 \leqslant j \leqslant k-1$, with $j$ odd.

Case 3: $B_{\ell}=A_{k-j-1}$ is in the $j$ th subdiagonal, for $0 \leqslant j \leqslant k-1$, with $j$ even.

Case 4: $B_{\ell}=A_{k-j-1}$ is in the $j$ th subdiagonal, for $0 \leqslant j \leqslant k-1$, with $j$ odd.

By Lemma 4.5, $B_{\ell}$ is the only nonzero block entry in the $j$ th subdiagonal. Moreover, (see Theorem 2.5), the block entries in the $i$ th subdiagonal, for $i=0, \ldots, k-1$, with $i \neq j$, are either: (i) $\lambda A_{k-i}$, (ii) $A_{k-i-1}$, (iii) $A_{k-i-1}+\lambda A_{k-i}$, or (iv) 0 .

Case 2: In this case, the $j$ coefficients $A_{k}, A_{k-1}, \ldots, A_{k-j+1}$ are located in the subdiagonals from 0 th to $(j-1)$ th. Since $j$ is odd, at least another coefficient must be unpaired, so the pencil is not sparse.

Case 3: Now there are $j+1$ coefficients $A_{k}, A_{k-1}, \ldots, A_{k-j}$ among the subdiagonals from 0th to $(j-1)$ th. Since $j$ is even, the pencil is, again, not sparse.

This proves the first part of the statement. The second part follows from Theorem 2.5. together with Lemma 4.4 and Lemma 4.6 In particular, conditions (i)-(iv) above determine the pattern of zero/nonzero subdiagonals, taking into account Lemma 4.4 . This is summarized in Table 1 


\begin{tabular}{|c|c|c|c|c|c|c|c|c|}
\hline \multirow{2}{*}{$\begin{array}{l}\text { Forms of the } \\
\text { block entries }\end{array}$} & \multicolumn{8}{|c|}{ Subdiagonal } \\
\hline & 0th & \begin{tabular}{|l|l|}
1 st & $\cdots$ \\
\end{tabular} & $(j-1)$ th & $j$ th & $(j+1)$ th & & $(k-2)$ th & ( $(k-1)$ th \\
\hline Case 1 & $\overline{A_{k-1}+\lambda A_{k}}$ & \begin{tabular}{|l|}
0 \\
\end{tabular} & 0 & $\lambda A_{k-j}$ & $A_{k-j-2}+\lambda A_{k-j-1}$ & 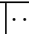 & 0 & $A_{0}+\lambda A_{1}$ \\
\hline Case 4 & $A_{k-1}+\lambda A_{k}$ & 0 & $A_{k-j}+\lambda A_{k-j+}$ & $A_{k-j-1}$ & 0 & . & 0 & $A_{0}+\lambda A_{1}$ \\
\hline
\end{tabular}

Table 1: Possible forms of the block entries in the subdiagonals for Cases 1 and 4.

Remark 4.9. Note that if $L_{1}(\lambda)$ and $L_{2}(\lambda)$ are two sparse pencils in $\mathscr{Q} \mathscr{C}_{n, k}$ of even degree $k$ such that $L_{1}(\lambda)$ has a monomial in the $j$ th subdiagonal (with $j$ even) of the form $B_{\ell}=\lambda A_{k-j}$ and $L_{2}(\lambda)$ has a monomial in the $(j+1)$ th subdiagonal of the form $B_{\ell}=A_{k-j-2}$, for $j=0,2, \ldots, k-2$, then $L_{1}(\lambda)$ and $L_{2}(\lambda)$ have the same nonzero subdiagonals. This is straightforward to see looking at the indices of the nonzero subdiagonals in Lemma 4.8 just replacing, for the case $j$ odd, $j$ by $j+1$.

Example 4.10. In this example we show all possible patterns for sparse pencils in $\mathscr{Q} \mathscr{C}_{n, 4}$ (that is, for quartic $n \times n$ matrix polynomials). Following Lemma 4.8 the zero/nonzero pattern of the subdiagonals depends on the subdiagonal containing the monomial $B_{\ell}$. Let this subdiagonal be the $j$ th one, for $j=0,1,2,3$. Then:

(a) For $j=0,1$, the monomial is $B_{\ell}=\lambda A_{4}($ for $j=0)$ or $B_{\ell}=A_{2}($ for $j=1)$, and the nonzero subdiagonals are the ones with indices 0,1 and 3 in both cases.

(b) For $j=2,3$, the monomial is $B_{\ell}=\lambda A_{2}($ for $j=2)$ or $B_{\ell}=A_{0}($ for $j=3)$. The nonzero subdiagonals are the ones with indices 0,2 and 3 in both cases.

The patterns are shown in Table 2
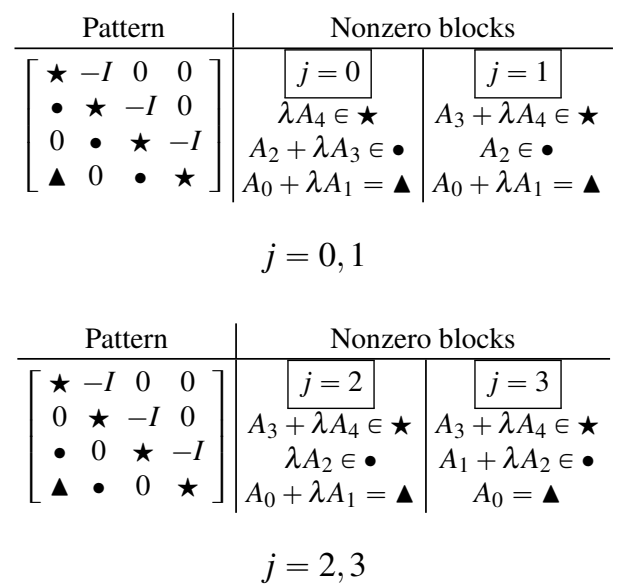

Table 2: All possible patterns for sparse pencils in $\mathscr{Q}_{\mathscr{C}_{n, 4}}$.

Since we know which are exactly the zero and nonzero subdiagonals in $\mathscr{Q} \mathscr{C}_{n, k}$, for $k$ odd and even, we can determine the number of different sparse pencils in 
$\mathscr{Q} \mathscr{C}_{n, k}$. We use, for a given $k \in \mathbb{N} \cup\{0\}$, the double factorial of $k$, defined by the recurrence relation: $k ! !:=(k-2) ! ! \cdot k$, if $k \geqslant 2$, and $k ! !:=1$ if $k \leqslant 1$.

Theorem 4.11. The number of different sparse pencils in $\mathscr{Q} \mathscr{C}_{n, k}$, for $k$ odd, is:

$$
\begin{aligned}
& 2\left(\sum_{j=1}^{\left\lfloor\frac{k}{4}\right\rfloor}((2 j-1) ! !)^{2}\left((2 j-1)^{\left\lceil\frac{k-4 j}{2}\right\rceil}+(2 j)^{\left\lceil\frac{k-4 j}{2}\right\rceil}\right)\right)+\left(\left(\frac{k-3}{2}\right) ! !\right)^{2}\left(\frac{k+1}{2}\right), \text { if }\left\lfloor\frac{k}{2}\right\rfloor \text { is even, and } \\
& 2\left(\sum_{j=1}^{\left\lfloor\left\lfloor\frac{k}{2}\right\rfloor / 2\right\rfloor}((2 j-1) ! !)^{2}\left((2 j-1)^{\left[\frac{k-4 j}{2}\right\rceil}+(2 j)^{\left\lceil\frac{k-4 j}{2}\right\rceil}\right)\right)+3\left(\left(\frac{k-1}{2}\right) ! !\right)^{2}, \text { if }\left\lfloor\frac{k}{2}\right\rfloor \text { is odd. }
\end{aligned}
$$

Proof. Let $k$ be an odd integer and let $1 \leqslant j \leqslant\left\lfloor\frac{k}{2}\right\rfloor$. We consider the rectangle $R_{j}$ of an $n k \times n k$ matrix pencil in $\mathscr{Q}_{1} \mathscr{C}_{n, k}$, whose vertices are ${ }_{k}(j, 1),(j, j),(k, 1),(k, j)$.

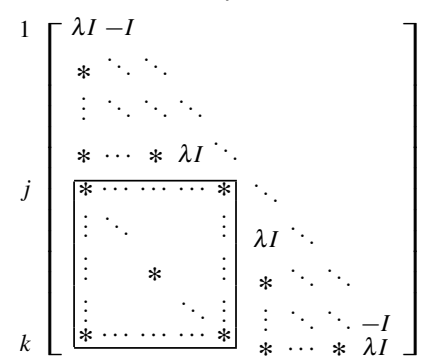

There are $\frac{k+1}{2}$ nonzero blocks among the asterisks $*$. By Lemma 4.7, they are of the form $A_{k-i-1}+\lambda A_{k-i}$, located in the $i$ th subdiagonal, for $i=0,2, \ldots, k-1$. We look for the number of different ways to place them in $R_{j}$.

This is summarized in Table 3, where we indicate the number of possible positions of each nonzero block in $R_{j}$. We need to take into account the parity of $j$.

Then, the number of possible sparse pencils in $\mathscr{Q} \mathscr{C}_{n, k}$, for $k$ odd, with all entries in $R_{j}$ is determined by counting all possible locations for the coefficients in Table 3.

In particular, if $\eta_{n, k}^{(j)}$ denotes the number of possible sparse pencils in $\mathscr{Q} \mathscr{C}_{n, k}$ with all entries in the rectangle $R_{j}$, then:

$$
\eta_{n, k}^{(j)}=\left\{\begin{array}{l}
1^{2} \cdot 3^{2} \cdot 5^{2} \cdots(j-1)^{2} \cdot(j)^{\left\lceil\frac{k-2 j}{2}\right\rceil}=((j-1) ! !)^{2}(j)^{\left\lceil\frac{k-2 j}{2}\right\rceil,} \quad \text { if } j \text { is even, and } \\
1^{2} \cdot 3^{2} \cdot 5^{2} \cdots(j-2)^{2} \cdot(j)^{\left\lceil\frac{k-2 j+2}{2}\right\rceil}=((j-2) ! !)^{2}(j)^{\left\lceil\frac{k-2 j+2}{2}\right\rceil,} \text { if } j \text { is odd } .
\end{array}\right.
$$

Now we consider all possible rectangles $R_{j}$, for $1 \leqslant j \leqslant\left\lfloor\frac{k}{2}\right\rfloor$. We only need to look at $j=1, \ldots,\left\lfloor\frac{k}{2}\right\rfloor$, and also at $j=\frac{k+1}{2}$, since for $j=\frac{k+3}{2}, \ldots, k$, the patterns are symmetric (with respect the anti-diagonal) to the first $\left\lfloor\frac{k}{2}\right\rfloor$ patterns. In the case $j=$ $\frac{k+1}{2}$, we can obtain the number of possible sparse pencils in $\mathscr{Q} \mathscr{C}_{n, k}$ with all its entries in the rectangle $R_{\frac{k+1}{2}}$ just replacing $j$ by $\frac{k+1}{2}$ in $(9)$, and this number is equal to: 


\begin{tabular}{|c|c|}
\hline Coefficient & Can be placed in ... \\
\hline$\lambda A_{k}+A_{k-1}$ & 1 position (0th subdiagonal) \\
\hline$\lambda A_{k-2}+A_{k-3}$ & 3 positions ( 2 nd subdiagonal) \\
\hline$\vdots$ & $\vdots$ \\
\hline $\int \lambda A_{k-(j-2)}+A_{k-(j-1)}$, or & $\int j-1$ positions $((j-2)$ th subdiagonal $)$ if $j$ even, or \\
\hline$\lambda A_{k-(j-3)}+A_{k-(j-2)}$ & $\{j-2$ positions $((j-3)$ th subdiagonal $)$ if $j$ odd \\
\hline$\left\{\begin{array}{l}\lambda A_{k-j}+A_{k-(j+1)}, \text { or } \\
\lambda A\end{array}\right.$ & $\left\{\begin{array}{cl}j \text { positions ( } j \text { th subdiagonal) } & \text { if } j \text { even, or }\end{array}\right.$ \\
\hline$M A_{k-(j-1)}+A_{k-j}$ & ( J positions $((J-1)$ th subalagonal $)$ lJ J oad \\
\hline & (. . $\quad$ : \\
\hline$\left\{\lambda A_{j+1}+A_{j}\right.$, or & $\{j$ positions $((k-j-1)$ th subdiagonal $)$ if $j$ even, or \\
\hline$\lambda A_{j}+A_{j-1}$ & ( j positions $((k-j)$ th subdiagonal $) \quad$ if $j$ odd \\
\hline$\lambda A_{j-1}+A_{j-2}$, or & $\{j-1$ positions $((k-j+1)$ th subdiagonal $)$ if $j$ even, or \\
\hline$\lambda A_{j-2}+A_{j-3}$ & $j-2$ positions $((k-j+2)$ th subdiagonal $)$ if $j$ odd \\
\hline$\vdots$ & $\vdots$ \\
\hline$\lambda A_{3}+A_{2}$ & 3 positions $((k-3)$ th subdiagonal $)$ \\
\hline$\lambda A_{1}+A_{0}$ & 1 position $((k-1)$ th subdiagonal $)$ \\
\hline
\end{tabular}

Table 3: Number of positions where each nonzero block entry can be in $R_{j}$

$$
\eta_{n, k}^{\left(\frac{k+1}{2}\right)}= \begin{cases}\left(\left(\frac{k-1}{2}\right) ! !\right)^{2}, & \text { if } \frac{k+1}{2} \text { is even, and } \\ \left(\left(\frac{k-3}{2}\right) ! !\right)^{2}\left(\frac{k+1}{2}\right), & \text { if } \frac{k+1}{2} \text { is odd } .\end{cases}
$$

Adding up, the total number of sparse pencils in $\mathscr{Q} \mathscr{C}_{n, k}$ is:

$$
\left\{\begin{array}{l}
2\left(\sum_{j=1}^{\left\lfloor\frac{k}{2}\right\rfloor / 2}\left(\eta_{n, k}^{(2 j-1)}+\eta_{n, k}^{(2 j)}\right)\right)+\eta_{n, k}^{\left(\frac{k+1}{2}\right)}, \quad \text { if }\left\lfloor\frac{k}{2}\right\rfloor \text { is even, and } \\
2\left(\sum_{j=1}^{\left\lfloor\left\lfloor\frac{k}{2}\right\rfloor / 2\right\rceil} \eta_{n, k}^{(2 j-1)}+\sum_{j=1}^{\left\lfloor\left\lfloor\frac{k}{2}\right\rfloor / 2\right\rfloor} \eta_{n, k}^{(2 j)}\right)+\eta_{n, k}^{\left(\frac{k+1}{2}\right)}, \text { if }\left\lfloor\frac{k}{2}\right\rfloor \text { is odd }
\end{array}\right.
$$

Using (9) and (10), and grouping summands appropiately in (11), we get (8).

Theorem 4.12. The number of different sparse pencils in $\mathscr{Q} \mathscr{C}_{n, k}$, for $k$ even, is:

$$
\begin{aligned}
& \frac{2}{3}\left(\sum_{j=1}^{\left\lfloor\frac{k}{4}\right\rfloor}((2 j-1) ! !)^{2}(3 k-4 j+4)\left((2 j-1)^{\frac{k-4 j+2}{2}}+(2 j)^{\frac{k-4 j+2}{2}}\right)\right), \text { if }\left\lfloor\frac{k}{2}\right\rfloor \text { is even, and } \\
& \frac{2}{3}\left(\sum_{j=1}^{\left\lfloor\frac{k}{4}\right\rfloor}((2 j-1) ! !)^{2}(3 k-4 j+4)\left((2 j-1)^{\frac{k-4 j+2}{2}}+(2 j)^{\frac{k-4 j+2}{2}}\right)\right)+ \\
& +\frac{2}{3}\left((2 k+2)\left(\left(\frac{k}{2}\right) ! !\right)^{2}\right), \text { if }\left\lfloor\frac{k}{2}\right\rfloor \text { is odd. }
\end{aligned}
$$


We will use the following lemma in the proof of Theorem 4.12 .

Lemma 4.13. Let $j$ be an integer. The following identities hold:

a) $\frac{(j-2) ! !}{(j-1) ! !} \sum_{i=0}^{\frac{j-4}{2}} \frac{(2 i+1) ! !}{(2 i) ! !}=\frac{j-2}{3}$, if $j \geqslant 4$ is an even number.

b) $\frac{(j-1) ! !}{(j-2) ! !} \sum_{i=0}^{\frac{j-3}{2}} \frac{(2 i+1) ! !}{(2 i) ! !}=\frac{j(j-1)}{3}$, if $j \geqslant 3$ is an odd number.

Proof. We divide the proof in two cases, depending on the parity of $j$.

a) If $j$ is an even number:

$$
\begin{aligned}
& \frac{(j-2) ! !}{(j-1) ! !} \sum_{i=0}^{\frac{j-4}{2}} \frac{(2 i+1) ! !}{(2 i) ! !}=\frac{2 \cdot 4 \cdot 6 \cdots(j-2)}{3 \cdot 5 \cdot 7 \cdots(j-1)}+\frac{4 \cdot 6 \cdots(j-2)}{5 \cdot 7 \cdots(j-1)}+\cdots+\frac{(j-4)(j-2)}{(j-3)(j-1)}+\frac{j-2}{j-1}= \\
& =\frac{j-2}{(j-1) ! !}(2 \cdot 4 \cdot 6 \cdots(j-4)+3 \cdot 4 \cdot 6 \cdots(j-4)+3 \cdot 5 \cdot 6 \cdots(j-4)+\cdots+ \\
& 3 \cdot 5 \cdots(j-5)(j-4)+3 \cdot 5 \cdots(j-5)(j-3))=\frac{j-2}{(j-1) ! !}(5 \cdot 4 \cdot 6 \cdots(j-4)+3 \cdot 5 \cdot 6 \cdots(j-4) \\
& +\cdots+3 \cdot 5 \cdots(j-5)(j-4)+3 \cdot 5 \cdots(j-5)(j-3))=\frac{j-2}{(j-1) ! !}(5 \cdot 7 \cdot 6 \cdots(j-4)+\cdots+ \\
& 3 \cdot 5 \cdots(j-5)(j-4)+3 \cdot 5 \cdots(j-5)(j-3))=\cdots+\frac{j-2}{(j-1) ! !}(5 \cdot 7 \cdots(j-1))=\frac{j-2}{3}
\end{aligned}
$$

b) If $j$ is an odd number:

$$
\begin{aligned}
& \frac{(j-1) ! !}{(j-2) ! !} \sum_{i=0}^{\frac{j-3}{2}} \frac{(2 i+1) ! !}{(2 i) ! !}=\frac{2 \cdot 4 \cdot 6 \cdots(j-1)}{3 \cdot 5 \cdot 7 \cdots(j-2)}+\frac{4 \cdot 6 \cdots(j-1)}{5 \cdot 7 \cdots(j-2)}+\cdots+\frac{(j-3)(j-1)}{(j-4)(j-2)}+\frac{j-1}{j-2}= \\
& =\frac{j-1}{(j-2) ! !}(2 \cdot 4 \cdot 6 \cdots(j-3)+3 \cdot 4 \cdot 6 \cdots(j-3)+3 \cdot 5 \cdot 6 \cdots(j-3)+\cdots+ \\
& 3 \cdot 5 \cdots(j-4)(j-3)+3 \cdot 5 \cdots(j-4)(j-2))=\frac{j-1}{(j-2) ! !}(5 \cdot 4 \cdot 6 \cdots(j-3)+3 \cdot 5 \cdot 6 \cdots(j-3) \\
& +\cdots+3 \cdot 5 \cdots(j-4)(j-3)+3 \cdot 5 \cdots(j-4)(j-2))=\frac{j-1}{(j-2) ! !}(5 \cdot 7 \cdot 6 \cdots(j-3)+\cdots+ \\
& 3 \cdot 5 \cdots(j-4)(j-3)+3 \cdot 5 \cdots(j-4)(j-2))=\cdots=\frac{j-1}{(j-2) ! !}(5 \cdot 7 \cdots(j-2) j)=\frac{j(j-1)}{3} .
\end{aligned}
$$

Proof (Theorem 4.12. Let $k$ be an even integer and let $1 \leqslant j \leqslant \frac{k}{2}$. We consider the rectangle $R_{j}$ with vertices $(j, 1),(j, j),(k, 1),(k, j)$, as in the proof of Theorem 4.11. First, we locate the nonzero block entry with only one coefficient, $B_{\ell}$, and, according to Lemma 4.8 , the remaining nonzero subdiagonals are uniquely determined.

We assume that $B_{\ell}=\lambda A_{k-i}$, which is in the $i$ th subdiagonal, for $i$ being an even number (case (a) in Lemma 4.8). For the other case $\left(B_{\ell}=A_{k-i-1}\right)$, the nonzero subdiagonals are exactly the same, by Remark 4.9 .

As in Theorem 4.11, we have to take into account the parity of the integer $j$ and the position of the monomial $B_{\ell}$. The procedure consists of counting, for each $B_{\ell}$, 
and for each $i$ even, the number of possible locations of each nonzero block entry inside $R_{j}$. It is also important to note that, in $R_{j}$,

$$
\text { the } i \text { th subdiagonal has } \begin{cases}i+1 \text { positions, } & \text { if } 0 \leqslant i \leqslant j-2, \\ j \text { positions, } & \text { if } j-1 \leqslant i \leqslant k-j, \text { and } \\ k-i \text { positions, } & \text { if } k-j+1 \leqslant i \leqslant k-1 .\end{cases}
$$

First, let us assume $j$ even. Then, depending on $i$ ( $i$ even), we obtain:

- If $i \leqslant j-4$ : The nonzero subdiagonals, by Lemma 4.8 , have indices $0,2,4, \ldots, i, i+$ $1, \ldots, j-3, j-1, \ldots, k-j-1, k-j+1, \ldots, k-3, k-1$, so, using (13), the number of possible locations for the nonzero blocks inside $R_{j}$ is:

$[1 \cdot 3 \cdot 5 \cdots(i+1)(i+2) \cdots(j-2)][j \cdots j][(j-1) \cdots 3 \cdot 1]=\left[(i+1) ! ! \frac{(j-2) ! !}{(i) ! !}\right]\left[j^{\frac{k-2 j+2}{2}}\right][(j-1) ! !]$.

- If $j-2 \leqslant i \leqslant k-j$ : The nonzero subdiagonals have indices $0,2, \ldots, j-$ $2, j, \ldots, i, i+1, \ldots, k-j-1, k-j+1, \ldots, k-3, k-1$, so the number of possible locations for the nonzero blocks inside $R_{j}$ is:

$$
[1 \cdot 3 \cdots(j-1)][j \cdots j \cdot j \cdots j][(j-1) \cdots 3 \cdot 1]=[(j-1) ! !]\left[j^{\frac{k-2 j+2}{2}}\right][(j-1) ! !] .
$$

- If $i \geqslant k-j+2$ : The nonzero subdiagonals have indices $0,2, \ldots, j-2, j, \ldots, k-$ $j, k-j+2, \ldots, i, i+1, \ldots, k-3, k-1$, so, using [13], the number of possible locations for the nonzero block entries inside $R_{j}$ is:

$$
\begin{aligned}
& {[1 \cdot 3 \cdots(j-1)][j \cdots j][(j-2) \cdots(k-i)(k-i-1) \cdots 3 \cdot 1]=} \\
& =[(j-1) ! !]\left[j^{\frac{k-2 j+2}{2}}\right]\left[(k-i-1) ! ! \frac{(j-2) ! !}{(k-i-2) ! !}\right] .
\end{aligned}
$$

Finally, if we denote by $E \eta_{n, k}^{(j)}$ the number of possible sparse pencils in $\mathscr{Q} \mathscr{C}_{n, k}$ with all entries in the rectangle $R_{j}$, for $j$ even, then, adding up all the above quantities, we get:

$$
\begin{gathered}
E \eta_{n, k}^{(j)}=2\left(\sum_{i=0}^{\frac{j-4}{2}}\left((2 i+1) ! ! \frac{(j-2) ! !}{(2 i) ! !}\right)\left(j^{\frac{k-2 j+2}{2}}\right)((j-1) ! !)+\sum_{i=\frac{j-2}{2}}^{\frac{k-j}{2}}((j-1) ! !)^{2}\left(j^{\frac{k-2 j+2}{2}}\right)+\right. \\
\left.+\sum_{i=\frac{k-j+2}{2}}^{\frac{k-2}{2}}((j-1) ! !)\left(j^{\frac{k-2 j+2}{2}}\right)\left((k-2 i-1) ! ! \frac{(j-2) ! !}{(k-2 i-2) ! !}\right)\right) .
\end{gathered}
$$

Note that the first and third summands in the last sum add up to the same number (just replace $i$ by $\frac{k-2}{2}-i$ in the sum of the third term). Moreover, the second summand does not depend on the index $i$. Then, $E \eta_{n, k}^{(j)}$ is equal to: 


$$
\begin{aligned}
E \eta_{n, k}^{(j)} & =4\left(j^{\frac{k-2 j+2}{2}}\right)((j-1) ! !)((j-2) ! !) \sum_{i=0}^{\frac{j-4}{2}} \frac{(2 i+1) ! !}{(2 i) ! !}+2((j-1) ! !)^{2}\left(j^{\frac{k-2 j+2}{2}}\right) \frac{k-2 j+4}{2}= \\
& \left.=((j-1) ! !)^{2}\left(j^{\frac{k-2 j+2}{2}}\right)\left[4 \cdot \frac{(j-2) ! !}{(j-1) ! !} \sum_{i=0}^{\frac{j-4}{2}} \frac{(2 i+1) ! !}{(2 i) ! !}+k-2 j+4\right] \text { (by Lemma } 4.13\right] \text { (a) ) } \\
& =((j-1) ! !)^{2}\left(j^{\frac{k-2 j+2}{2}}\right)\left[4 \cdot \frac{j-2}{3}+k-2 j+4\right]=((j-1) ! !)^{2}\left(j^{\frac{k-2 j+2}{2}}\right)\left(\frac{3 k-2 j+4}{3}\right) .
\end{aligned}
$$

Repeating this procedure for $j$ odd, if we denote by $O \eta_{n, k}^{(j)}$ the number of all possible sparse pencils in $\mathscr{Q} \mathscr{C}_{n, k}$ with all entries in the rectangle $R_{j}$, for $j$ odd, then:

$$
\begin{gathered}
O \eta_{n, k}^{(j)}=2\left(\sum_{i=0}^{\frac{j-3}{2}}\left((2 i+1) ! ! \frac{(j-1) ! !}{(2 i) ! !}\right)\left(j^{\frac{k-2 j+2}{2}}\right)((j-2) ! !)+\sum_{i=\frac{j-1}{2}}^{\frac{k-j-1}{2}}((j-2) ! !)^{2}\left(j^{\frac{k-2 j+4}{2}}\right)+\right. \\
\left.+\sum_{i=\frac{k-j+1}{2}}^{\frac{k-2}{2}}((j-2) ! !)\left(j^{\frac{k-2 j+2}{2}}\right)\left((k-2 i-1) ! ! \frac{(j-1) ! !}{(k-2 i-2) ! !}\right)\right) .
\end{gathered}
$$

As above, we can simplify $O \eta_{n, k}^{(j)}$ as:

$$
\begin{aligned}
O \eta_{n, k}^{(j)} & =4\left(j^{\frac{k-2 j+2}{2}}\right)((j-2) ! !)((j-1) ! !) \sum_{i=0}^{\frac{j-3}{2}} \frac{(2 i+1) ! !}{(2 i) ! !}+2((j-2) ! !)^{2}\left(j^{\frac{k-2 j+4}{2}}\right) \frac{k-2 j+2}{2}= \\
& \left.=((j-2) ! !)^{2}\left(j^{\frac{k-2 j+2}{2}}\right)\left[4 \cdot \frac{(j-1) ! !}{(j-2) ! !} \sum_{i=0}^{\frac{j-3}{2}} \frac{(2 i+1) ! !}{(2 i) ! !}+j(k-2 j+2)\right] \text { (by Lemma } 4.13(\text { b })\right) \\
& =((j-2) ! !)^{2}\left(j^{\frac{k-2 j+2}{2}}\right)\left[4 \cdot \frac{j(j-1)}{3}+j(k-2 j+2)\right]= \\
& =((j-2) ! !)^{2}\left(j^{\frac{k-2 j+2}{2}+1}\right)\left(\frac{3 k-2 j+2}{3}\right)=(j ! !)^{2}\left(j^{\frac{k-2 j}{2}}\right)\left(\frac{3 k-2 j+2}{3}\right)
\end{aligned}
$$

In summary,

$$
E \eta_{n, k}^{(j)}=((j-1) ! !)^{2}\left(j^{\frac{k-2 j+2}{2}}\right)\left(\frac{3 k-2 j+4}{3}\right), \quad O \eta_{n, k}^{(j)}=(j ! !)^{2}\left(j^{\frac{k-2 j}{2}}\right)\left(\frac{3 k-2 j+2}{3}\right) .
$$

Now, we consider all possible rectangles $R_{j}$, for $1 \leqslant j \leqslant \frac{k}{2}$. We just look at $j=$ $1, \ldots, \frac{k}{2}$, since for $j=\frac{k}{2}+1, \ldots, k$, the patterns are symmetric (with respect the antidiagonal) to the first ones. Adding up, the number of sparse pencils in $\mathscr{Q}_{n} n$ is:

$$
\left\{\begin{array}{l}
2\left(\sum_{j=1}^{\frac{k}{4}}\left(O \eta_{n, k}^{(2 j-1)}+E \eta_{n, k}^{(2 j)}\right)\right), \text { if } \frac{k}{2} \text { is even, and } \\
2\left(\sum_{j=1}^{\left\lceil\frac{k}{4}\right\rceil} O \eta_{n, k}^{(2 j-1)}+\sum_{j=1}^{\left\lfloor\frac{k}{4}\right\rfloor} E \eta_{n, k}^{(2 j)}\right), \text { if } \frac{k}{2} \text { is odd. }
\end{array}\right.
$$


Using (14) and grouping summands appropiately in (15), we arrive at (12).

Remark 4.14. Note that the number of sparse companion pencils in $\mathscr{Q} \mathscr{C}_{n, k}$ for $k$ even becomes much larger than the one for $k$ odd as $k$ increases. This is due to the fact that the nonzero subdiagonals in the case $k$ odd are determined (they are the ones with indices $0,2, \ldots, k-1)$, but the case $k$ even allows for more flexibility, depending on which is the nonzero subdiagonal containing the block $B_{\ell}$ (see Lemma 4.8.

\section{Conclusions}

In this work, we have first introduced a family of companion pencils for $n \times n$ matrix polynomials of degree $k$ over an arbitrary field, $\mathscr{R}_{n, k}$, which extends the one in [16] for companion matrices of monic scalar polynomials. This family contains all companion pencils in most of the families of companion linearizations introduced so far in the literature, expressed in the monomial basis, and having a small number of nonzero entries. In particular, $\mathscr{R}_{n, k}$ contains both Fiedler and generalized Fiedler pencils, as well as all sparse pencils in the block-Kronecker linearizations presented in [15]. We have provided a "canonical" expression for companion pencils in $\mathscr{R}_{n, k}$, up to block permutation. This expression, which leads to the class $\mathscr{Q}_{n, k}$, is block upper Hessenberg and resembles the one provided in [16] for companion matrices of monic scalar polynomials, We have provided a characterization for a pencil in $\mathscr{Q}_{n, k}$ to be a companion pencil (namely, they are those in the class denoted by $\mathscr{Q} \mathscr{C}_{n, k}$ ). Finally, we have obtained the number of different sparse companion pencils in $\mathscr{R}_{n, k}$, up to block permutation. We want to emphasize that there could be other sparse companion pencils for $n \times n$ matrix polynomials of degree $k$ not included in $\mathscr{R}_{n, k}$. Therefore, describing all sparse companion pencils for $n \times n$ matrix polynomials of degree $k$ is still an open field of research.

Acknowledgments. We wish to thank two anonymous referees for the careful reading of this paper and for their comments, that allowed us to improve the presentation.

This work has been partially supported by the Ministerio de Economía y Competitividad of Spain through grants MTM2015-68805-REDT and MTM2015-65798-P.

\section{References}

1. E. N. Antoniou, S. Vologiannidis. A new family of companion forms for polynomial matrices. Electron. J. Linear Algebra, 11 (2004) 78-87.

2. M. I. Bueno, K. Curlett, S. Furtado. Structured strong linearizations from Fiedler pencils with repetition I. Linear Algebra Appl., 460 (2014) 51-80.

3. M. I. Bueno, F. De Terán. Eigenvectors and minimal bases for some families of Fiedler-like linearizations. Lin. Multilin. Algebra, 62 (2014) 39-62. 
4. M. I. Bueno, F. De Terán, F. M. Dopico. Recovery of eigenvectors and minimal bases of matrix polynomials from generalized Fiedler linearizations. SIAM J. Matrix Anal. Appl., 32 (2011) 463-483.

5. M. I Bueno, F. M. Dopico, S. Furtado, M. Rychnovsky. Large vector spaces of blocksymmetric strong linearizations of matrix polynomials. Linear Algebra Appl., 477 (2015) 165-210.

6. M. I Bueno, F. M. Dopico, J. Pérez, R. Saavedra, B. Zykovsi. A unified approach to Fiedlerlike pencils via strong block minimal bases pencils. Linear Algebra Appl., 547 (2018) 45-104.

7. M. I. Bueno, S. Furtado. Palindromic linearizations of a matrix polynomial of odd degree obtained from Fiedler pencils with repetition Electron. J. Linear Algebra, 23 (2012) 562-577.

8. F. De Terán, F. M. Dopico, D. S. Mackey. Fiedler companion linearizations and the recovery of minimal indices. SIAM J. Matrix Anal. Appl., 31 (2010) 2181-2204.

9. F. De Terán, F. M. Dopico, D. S. Mackey. Palindromic companion forms for matrix polynomials of odd degree, J. Comput. Appl. Math., 236 (2011) 1464-1480.

10. F. De Terán, F. M. Dopico, D. S. Mackey. Fiedler companion linearizations for rectangular matrix polynomials. Linear Algebra Appl., 437 (2012) 957-991.

11. F. De Terán, F. M. Dopico, D. S. Mackey. Spectral Equivalence of matrix polynomials and the Index Sum Theorem. Linear Algebra Appl., 459 (2014) 264-333.

12. F. De Terán, F. M. Dopico, J. Pérez. Backward stability of polynomial root-finding using Fiedler companion matrices. IMA J. Numer. Anal. 36 (2016) 133-173.

13. F. De Terán, F. M. Dopico, J. Pérez. Eigenvalue condition number and pseudospectra of Fiedler matrices. Calcolo, 54 (2017) 319-365.

14. G. Del Corso, F. Poloni. Counting Fiedler pencils with repetition. Linear Algebra Appl., 532 (2017) 463-499.

15. F. M. Dopico, P. Lawrence, J. Pérez, P. Van Dooren. Block Kronecker linearizations of matrix polynomials and their backward errors. To appear in Numer. Math. Available as MIMS Eprint 2016.34. The University of Manchester. UK.

16. B. Eastman, I.-J. Kim, B. L. Shader, K. N. Vander Meulen. Companion matrix patterns. Linear Algebra Appl., 436 (2014) 255-272.

17. B. Eastman, K. N. Vander Meulen. Pentadiagonal companion matrices. Spec. Matrices, 4 (2016) 13-30.

18. C. Garnett, B. L. Shader, C. L. Shader, P. van den Driessche. Characterization of a family of generalized companion matrices. Linear Algebra Appl. 498 (2016) 360-365.

19. C. Ma, X. Zhan. Extremal sparsity of the companion matrix of a polynomial. Linear Algebra Appl., 438 (2013) 621-625.

20. V. Noferini, J. Pérez. Fiedler-comrade and Fiedler-Chebyshev pencils. SIAM J. Matrix Anal Appl., 37 (2016) 1600-1624.

21. V. Noferini, J. Pérez. Chebyshev rootfinding via computing eigenvalues of colleague matrices: when is it stable? Math. Comp. 86 (2017) 1741-1767.

22. L. Robol, R. Valdebril, P. Van Dooren. A framework for structured linearizations of matrix polynomials in various bases. SIAM J. Matrix Anal. Appl., 38 (2017) 188-216.

23. S. Vologiannidis, E. N. Antoniou. A permuted factors approach for the linearization of polynomial matrices. Math. Control Signals Syst., 22 (2011) 317-342. 